\title{
Archéopages
}

Archéopages

Archéologie et société

40 | 04-07/2014

Villages

\section{La céramique à Acy-Romance et dans le pays rème (vers 300 à $30 \mathrm{av.} \mathrm{J.-C.)}$}

Descriptions et hypothèses fonctionnelles

\section{Marion Saurel}

\section{OpenEdition}

\section{Journals}

Édition électronique

URL : https://journals.openedition.org/archeopages/669

DOI : $10.4000 /$ archeopages. 669

ISSN : 2269-9872

\section{Éditeur}

INRAP - Institut national de recherches archéologiques préventives

Édition imprimée

Date de publication : 1 juin 2015

Pagination : 177-178

ISSN : 1622-8545

\section{Référence électronique}

Marion Saurel, « La céramique à Acy-Romance et dans le pays rème (vers 300 à 30 av. J.-C.) », Archéopages [En ligne], 40 | 04-07/2014, mis en ligne le 01 juillet 2016, consulté le 23 janvier 2022 URL : http://journals.openedition.org/archeopages/669; DOI : https://doi.org/10.4000/archeopages. 669 
panorama des aménagements provisoires du chantier où, encore une fois, le bois joue un rôle prépondérant. Couplées avec l'analyse des parements, ces structures provisoires permettent d'identifier des séquences de montage des maçonneries, des ruptures dans le travail ou des changements de projet.

À travers la synthèse de ces données, il a été possible de toucher aux aspects sociaux du chantier dont l'organisation du travail et des éventuelles expressions de l'individualité des ouvriers ou d'une équipe. Les techniques de montage et l'interprétation des marques lapidaires posent la question de la mobilité des artisans et la transmission du savoir-faire, mais cet aspect du chantier a sans doute été insuffisamment développé. La dimension humaine reste moins facilement abordable que les aspects techniques. Sans doute faudrait-il d'autres données pour mieux cerner les bâtisseurs restés encore en filigrane. La question du cadre de vie a été abordée par le biais des observations sur les éléments de finition comme les revêtements au sol, les enduits, les éléments de confort et d'hygiène. Les aléas de la conservation ont toutefois tendance à nous laisser avec l'impression d'une coquille vide alors que le bâti ancien sous toutes ses formes était garni par le second œuvre des différents corps de métier et par un mobilier parfois riche. Cet aspect de notre travail, et l'appréciation du « comment vivre» méritent également d'être approfondis.

En appréhendant le chantier dans sa matérialité, l'approche archéologique permet d'écrire une histoire de la construction parfois bien différente quoique complémentaire à celle connue de l'analyse stylistique ou de l'étude des textes. L'exploitation de ces données démontre que l'archéologie représente une véritable plus-value non seulement pour les besoins de la gestion du patrimoine architectural, mais aussi pour la recherche scientifique. Elle a également démontré tantôt par sa présence tantôt par son absence, l'importance du concours des autres disciplines et d'un regard pluriel pour passer du stade de la mise en phase et de la description, aussi complètes soient-elles, à celui d'une interprétation approfondie: historiens de l'art, de l'architecture et de la religion, les spécialistes des matériaux, ethnologues et hommes de métier. Le chantier de restauration ou d'aménagement reste toujours un lieu privilégié où sont réunis les moyens matériels et humains et la possibilité d'accéder à notre objet d'étude. Les méthodologies mises en œuvre dans ce contexte sont certes puisées dans le répertoire commun à tous les archéologues. Cependant, les enjeux qui lui sont propres nécessitent encore de trouver des réponses institutionnelles pour améliorer la prise en compte de notre discipline.
Marion Saurel

Inrap, UMR 8546

8 AVRIL 2014

Université Paris I, PanthéonSorbonne, École doctorale d'archéologie (ED 112)

Directeur de thèse :

Jean-Paul Demoule,

Professeur, Université Paris I

Membres du Jury:

Rapporteurs:

Philippe Barral,

Professeur, Université

de Franche-Comté

Marie-Yvane Daire,

Directrice de recherche, CNRS,

Experts :

Bernard Lambot,

Archéologue bénévole

Jeannot Metzler,

Conservateur, MNHA Luxembourg

SAUREL M., à paraître: La céramique à Acy-Romance et dans le pays rème (vers 300 à 30 avant J.C.) descriptions et hypothèses fonctionnelles, in Mémoires de la Société archéologique champenoise (Monographie du site protohistorique d'AcyRomance (Ardennes); VI).
«Archéologie et philologie d'Orient et d'Occident »

\section{La céramique à Acy-Romance et dans le pays rème (vers 300 à $\mathbf{3 0}$ av. J.-C.).}

\section{Descriptions et hypothèses fonctionnelles.}

L'apport de la céramique à la connaissance des pratiques sociales de lâge du Fer passe par une approche concrète de l'utilisation des récipients et ustensiles. Si l'expérimentation et les analyses chimiques, notamment de matières biologiques, en plein développement, sont appelées à jouer un rôle décisif, elles sont indissociables d'une étude fonctionnelle systématique des restes céramiques intégrant l'ensemble des données - la morphologie, la technique, la capacité, les traces, mais aussi les contextes et l'environnement au sens large.

Les principaux corpus traités sont issus d'une part, de la fouille programmée d'Acy-Romance dirigée par Bernard Lambot et d'autre part, de fouilles préventives de l'Inrap sur le parcours du TGV Est. Les sites appartiennent au nord de la plaine crayeuse de Champagne, entre le cours de l'Aisne que surplombe le village d'Acy-Romance occupé aux IIe-Ier siècle avant notre ère et la vallée de la Vesle où se trouvent les habitats de Champfleury et de Villers-aux-Nœuds, datés des IVe-IIe siècles. Ils s'inscrivent au cœur de ce qui fut à la fin de l'âge du Fer le domaine des Rèmes quelles que soient les fluctuations envisagées pour cette entité territoriale.

Le premier volet, consacré à la périodisation de la céramique d'Acy-Romance dans son cadre régional, permet de cerner l'évolution de la production céramique et de replacer les phénomènes dans une perspective historique. Les logiques internes à cette évolution recoupent les phases de l'histoire du village du premier temps d'inventivité foisonnante autour du milieu du Ile siècle avant notre ère en accord avec une agglomération dynamique, à une période de basculement puis à une standardisation des procédés et des formes concomitantes au développement des oppida et à la perte d'importance du site à l'échelle du pays rème. Cette réflexion repose sur deux classements morphologique et technique - élaborés de manière à s'accorder avec l'approche fonctionnelle et apporte une maîtrise relative de la donnée « temps » qui constitue un préliminaire indispensable.

La première étape est celle de la mise en place des outils spécifiques à l'analyse fonctionnelle; tout d'abord, le classement des récipients et ustensiles du corpus en «familles» (d'après la technique, la morphologie et la capacité) et, en second lieu, le classement raisonné des traces liées à l'emploi. Chaque famille et chaque catégorie de traces font l'objet d'un bilan documentaire afin de déterminer un premier éventail des possibles. Deuxièmement, le croisement des données correspond à une analyse systématique de la représentation des traces pour chaque famille, et au recoupement des 
résultats entre les familles afin de nourrir l'argumentation et de dégager des pistes de réflexion. Le troisième temps est celui de la discussion autour des hypothèses d'utilisation en faisant appel à des sources variées, études archéologiques et ethnographiques, textes antiques ou traités techniques.

Le croisement des familles et des traces, effectué pour plus de 1400 céramiques, a fait émerger plusieurs liens dont certains permettent de proposer de nouvelles pistes interprétatives. Des stigmates comparables sur les jarres (dont les dolia) et sur les formes fermées les plus fines consistent en particulier dans des signes d'altération des surfaces intérieures par le contenu: porosité «moldique» (disparition des inclusions calcaires) et lacunes en cupules. Les autres liens entre ces familles fonctionnelles sont les signes d'usure de la lèvre attestant de l'emploi de couvercles et le profil qui tend à se resserrer à l'embouchure. Par contre, par d'autres traits, les jarres se rapprochent de la vaisselle culinaire et un usage au feu peut être envisagé. Les comparaisons dans le premier âge du Fer, la nature des traces, les données environnementales avec la prédominance de la culture de l'orge dans la région et des témoignages ethnoarchéologiques sur les céramiques à boisson fermentée sont autant d'arguments pour envisager un emploi des grandes jarres à la surface altérée pour la cuisson et/ou la fermentation de la bière d'orge.

Le lien presque exclusif entre dépôt blanchâtre intérieur (de nature calcaire d'après les analyses) et fond à large(s) perforation(s) se prête à un examen minutieux des causes potentielles à travers les formes respectives des ustensiles et du dépôt. Après élimination successive, la réflexion s'est concentrée autour d'une hypothèse d'emploi de ces céramiques à fond perforé comme entonnoir pour la fabrication de l'eau de chaux. Les perspectives ouvertes par cette hypothèse sont multiformes. Les découvertes régionales suggèrent que cette pratique fut répandue dans la société rème des IIIe et IIe siècles avant notre ère. Différents indices permettent de la replacer dans le cadre du développement d'une production à grande échelle de la chaux, aux usages multiples, développement qui n'est vraisemblablement pas étranger à la prédilection des Gaulois pour une implantation sur les versants et sommets calcaires où la ressource était aisément exploitable.

Létude fonctionnelle des restes céramiques en contextes archéologiques permet d'apporter des questionnements particuliers amenés à nourrir une réflexion qui ne peut être au-delà que pluridisciplinaire. Elle passe par la prise en compte de l'environnement physique (composition des pâtes, nature et conservation des traces...) et culturel (maîtrise technologique, pratiques agricoles...).

Faire émerger ces questions implique de se garder d'appliquer un cadre trop théorique; les familles «fonctionnelles» de récipients et ustensiles doivent ressortir de l'analyse interne des corpus et garder des contours souples destinés à évoluer. Cela paraît particulièrement nécessaire pour la céramique de production peu spécialisée des régions du nord de la Gaule jusquà la phase finale de l'âge du Fer, pour laquelle l'appréhension de traits fonctionnels passe par la perception d'infinies nuances. 